\title{
Erratum: Proton Therapy Review: Proton Therapy from a Medical
}

\section{Se Byeong Lee ${ }^{(1)}$}

Department of Radiation Oncology \& Proton Therapy Center, National Cancer Center, Goyang, Korea

Progress in Medical Physics 31(3), September 2020

https://doi.org/10.14316/pmp.2020.31.3.99

In the originally published version of this article, there were some missing words in the Title, which sould be properly revised as follows:

\section{Before correction:}

Proton Therapy Review: Proton Therapy from a Medical

\section{After correction:}

Proton Therapy Review: Proton Therapy from a Medical Physicist's View

We apologize for our mistake and any inconvenience this may have caused. 